flächenhaft anhaftenden Wassers, 3) die Versuche von Hoppe nicht zahlreich genug sind ,um nicht hie und da Einiges zweifelhaft zu lassen".

Es erübrigt mir nur noch, meinem hochverehrten Lehrer, Herrn Hofrath Professor Preyer meinen Dank auszusprechen für die grosse Liebenswürdigkeit, mit welcher er mich stets durch Rath und That unterstützte.

Jena, Physiologisches Institut, im Juli 1888.

\title{
VIII.
}

\section{Ueber eine cystische Geschwulst der Leber (Gallengangscystadenom).}

\author{
Von Arnold Siegmund, 7. Z. in Göttingen. \\ (Hierzu Taf. III-IV.)
}

Im December 1887 wurde dem Göttinger pathologischen Institute von Herrn Dr. Reichelt in Hannover eine Leber mit cystischem Tumor übersandt, welche Herr Professor Dr. Orth die Güte hatte, mir zur Bearbeitung zu überweisen.

Die Mittheilungen des Herrn Dr. Reichelt lauten so:

„Frau S., 65 Jahre alt, hat 9 Kinder leicht geboren, das letzte vor etwa 20 Jahren. Seitdem bemerkte sie, dass der Leib stärker blieb, als es sonst der Fall war. Seit jener Zeit hat sie auch stets an hartem Stuhl gelitten. Dass derselbe jemals thonfarben gewesen sei, bestreitet sie bestimmt; Medicamente hat sie nie genommen. Schmerzen hat ihr die Geschwulst nie bereitet, sie ist bis zuletzt eine starke Person gewesen, die immer schwer arbeiten konnte. Krank ist sie nach Aussage ibres Nannes nie gewesen, ausser vor $7 \mathrm{Jahren,}$ wo sio wegen Appetitlosigkeit, Kopfschmerzen und Schmerzen in der Lebergegend 6 Wochen zu Bett lag. Sie wurde wieder so gesund, wie vorher. Sie hat immer behauptet, dass seit jener Zeit der Tumor nicht weiter gewachsen sei."

"Zuletzt ist sie etwa 4 Wochen krank gewesen; ich sab sie zum ersten Male etwa 30 Stunden vor ihrem Tode. Nach langen Verhandlungen wurde mir gestattet, die Bauchsection zu machen und dazu nur eine halbe Stunde Zeit gewährt."

"Die Bauchorgane waren sämmtlich gesund - makroskopisch! - Nur die ungemein vergrösserte Leber mit der Oystenbildung fiel auf. Der Tumor 


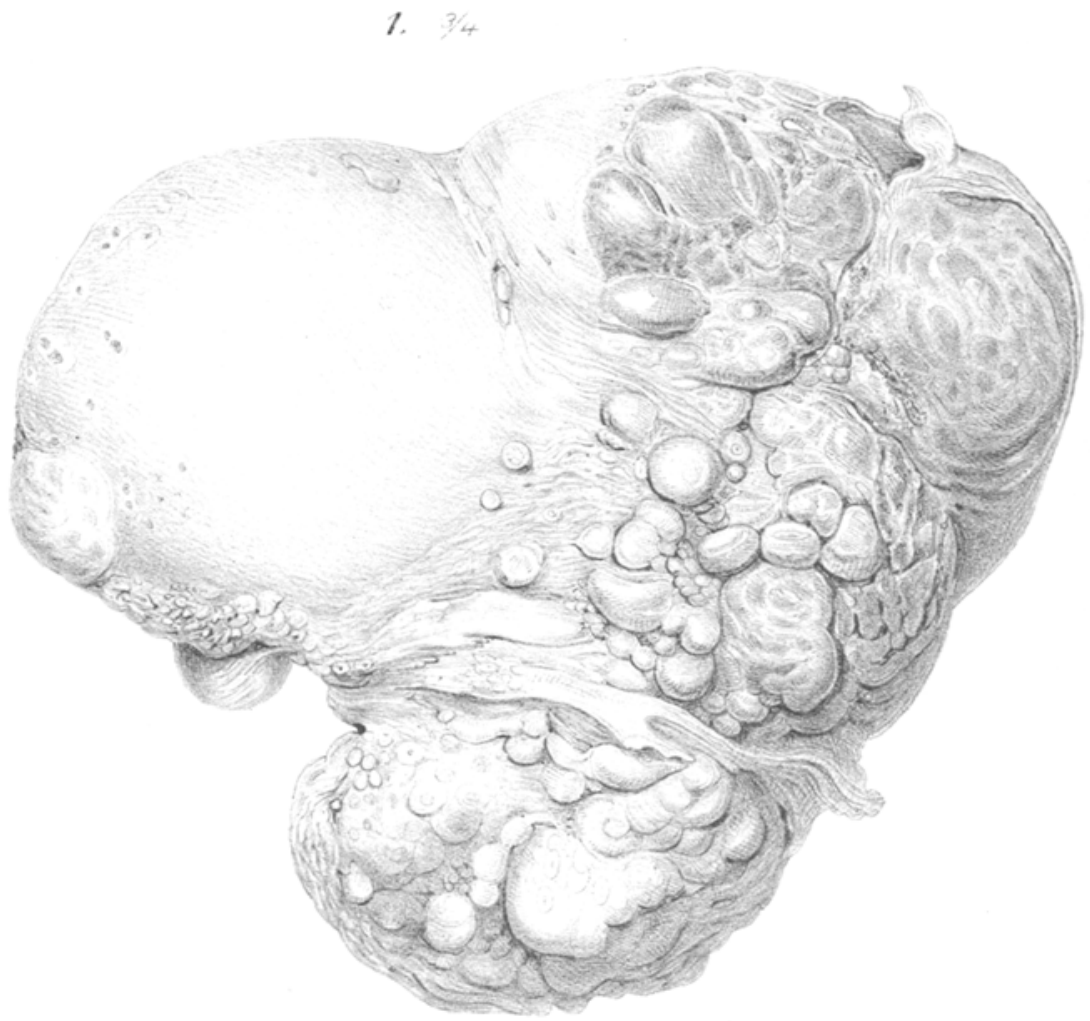

2

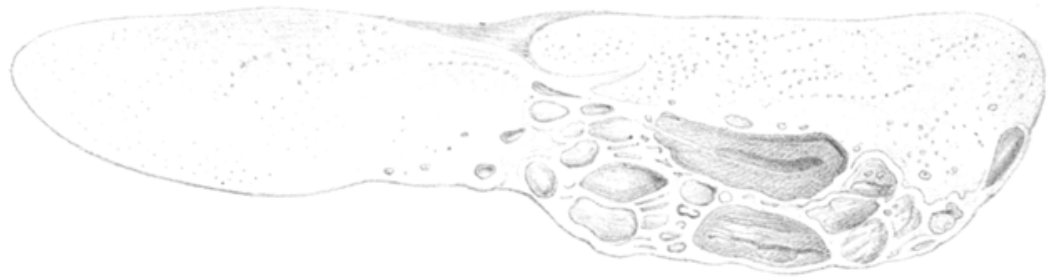



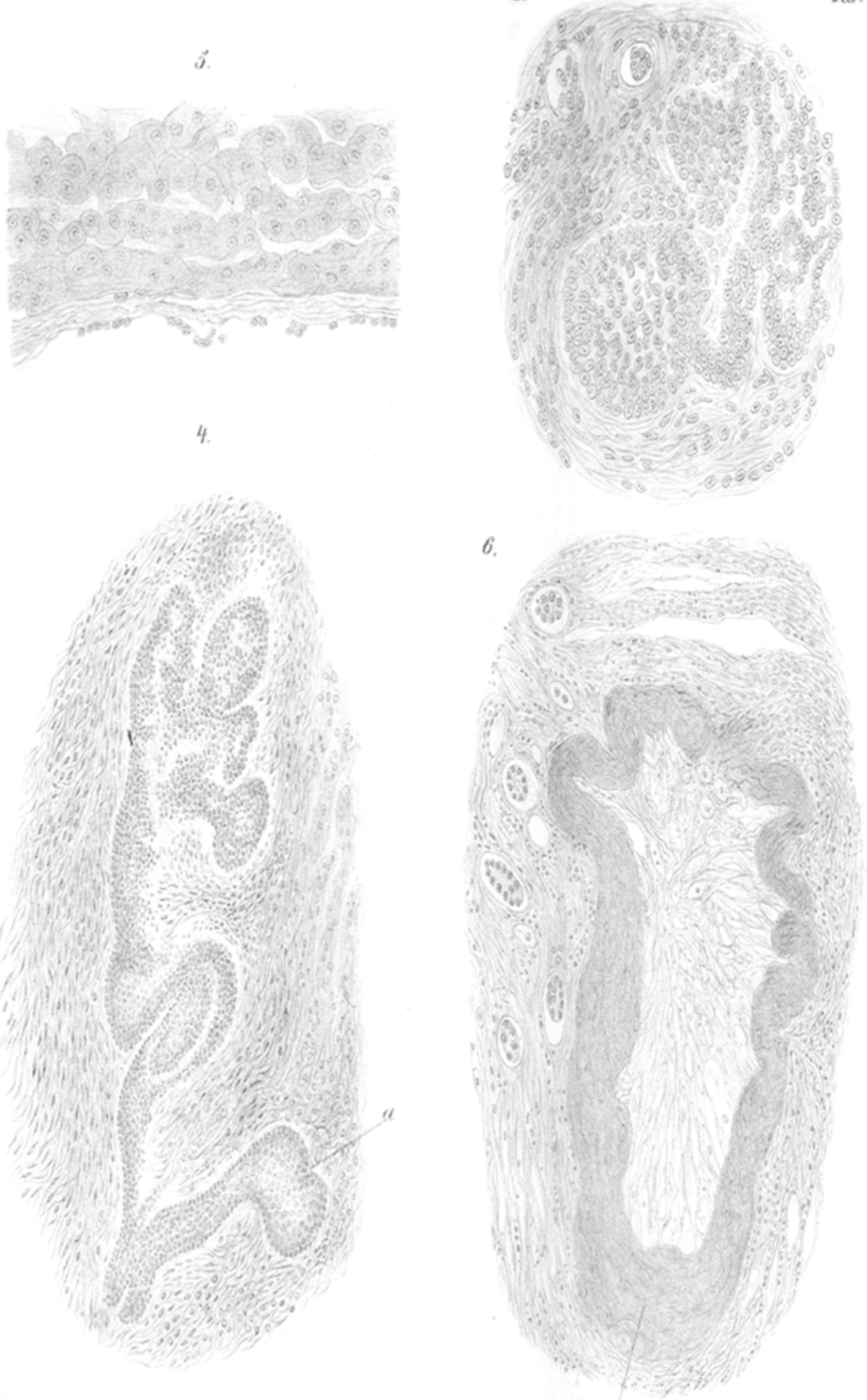


\section{6}

war an seiner ganzen Oberfläche frei von Verwachsungen, nur die untere Fläche war adhärent und die Verwachsungen mussten mit dem Messer getrennt werden."

Von der übersandten Leber habe ich folgenden Sectionsbefund aufgenommen:

An der Leber (Fig. 1) fällt ein grosser, aus vielen Cysten bestehender Tumor auf, welcher reichlich die Hälfte ibrer Oberfläche einnimmt und so, zumal er sämmtliche freie Ränder des von ihm besetzten Theiles überragt, die Gestalt des Organes völlig verändert. Man siebt an dem vom Tumor freigelassenen Theile der Leber zwei lauge Ränder, einen ganz scharfen, welcher mit einer Kerbe, gebildet durch Zusammeufluss zweier flacher, in der Vorder- und Hinterfläche befindlicher Furchen versehen ist, und einen dickeren, unter dem sich der Grund der Gallenblase bervorwölbt. An der Vorderfläche zieht von der Gegend der letzteren zur Vena cava quer über den Tumor hinweg das Lig. suspensorium, die Grenzen der beiden grossen Leberlappen bestimmend. Diese baben nicht das gewöhnliche Grössenverbältniss, vielmehr ist der linke viel zu klein, der rechte bedeutend $z u$ gross.

Aus dem parallelen Verlauf der genannten Ränder zu dem Lig. susp. ergiebt sich, dass diese die seitlichen Grenzen des rechten Leberlappens darstellen, welcher eine Gestaltveränderung erfahren hat, die ihrerseits die Lage der Gallenblase beeinflusste.

Diese merkwürdige Veränderung der Gestalt des rechten Leberlappens ist, da er am meisten von der Cystenentwickelung betroffen ist, möglicherweise als die Folge einer vicariirenden Hypertrophie aufzufassen, oder besser, da die Leberläppchen nicht vergrössert zu sein scheinen, als Folge einer Neubildung von Lobuli; doch spricht die Einkerbung an dem rechten Rande des Lappens auch für die Betheiligung mechaniseher Einwirkungen an der Gestaltung desselben. (Schnürleber).

Der Tumor nimmt, da er sich auf beiden Seiten vom Lig. susp. ausbreitet, auf der Vorderseite der Leber den linken Lappen ganz und dazu einen grossen Theil des rechten ein. Das Aeussere des Organs giebt die Abbildung No. 1 sehr gut wieder. Die Zeichnung des sagittalen Durchschnittes (Fig. 2) durch den rechten Lappen lässt erkennen, dass der von cystischer Entartung befallene Theil desselben auch im Innern, und zwar bis in die Mitte, von Cysten durchsetzt wird.

Das Gewicht der Leber beträgt $2610 \mathrm{~g}$, die Höhe des linken Lappens $5 \mathrm{~cm}$, des rechten 9 bezw. $7 \frac{1}{2} \mathrm{~cm}$, die Dicke des linken ist $12 \frac{1}{2} \mathrm{~cm}$, die des rechten $26 \mathrm{~cm}$. Der horizontale Durchmesser ist $35 \mathrm{~cm}$ lang.

Die äusserst zahlieichen Cysten der Geschwulst zeigen alle Grössen zwischen der von Linsen und der von Hühnereiern, sind aber durchschnittlich nur kirsch- bis pflaumengross. Sie sind durch bindegewebige Wände von einander getrenut, in denen das blosse Ange kein Lebergewebe erkennt, sind prall gespannt durch Flüssigkeit, haben aussen glänzende, etwas durchscheinende, glatte, bei manchen grün und roth gefärbte Wandungen, die viel dänner und auch nicht so elastisch sind, wie die von Echinokokken- 


\section{7}

blasen. Die innere Wandseite ist ebenso. Leisten, welche auf Vereiuigung kleiner Cysten zu einer grossen deuten könnten, fehlten.

In dern vom Tumor freigelassenen Lebertheil, dessen Farbe braun, dessen Festigkeit verstärkt, dessen Obertläche glatt und dessen Lobuli deutlich sichtbar und anscheinend von gewöhnlicher Grösse sind, sitzt noch eine Anzahl zerstreuter Cysten von Linsengrösse und darunter; einige sind mit blossem Auge eben noch erkennbar, andere erreichen Wallnussgrösse. In der hinteren Leberfäche sind gleichfalls einige $\mathrm{za}$ bemerken, besonders in der Gegend des Lig. triangulare. Die ganze Unterseite zeigt viele durchschnittene Verwachsungen.

Beim Anschneiden entleeren die Cysten eine trübe gelbliche Flüssigkeit, welche einen flockigen Bodensatz ausfallen lässt. Sie ist dünnflüssig, nicht fadenziehend, und reagirt schwach alkalisch. Sie giebt mit Alcohol absolutus einen gelblich-weissen, in Wasser löslichen, mit Eisessig einen weisslichen, im Ueberschuss der Säure unlöslichen Niederschlag, welcher unter dem $\mathrm{Hi}$ kroskop als eine feinkörnige Masse erscheint. Die Flüssigkeit ist demnach mucinhaltig.

Die Ginelin'sche Probe (Salpetersäure + etwas rauchender Salpetersäure) ergab keine Gallenfarbstoffreaction. Dagegen entstand ein massiger Niederschlag von Eiweiss, der möglicherweise vorhandene Gallenfarbstoffe mit zu Boden gerissen und so die Probe gestört hat.

Der beim Stehen ausfallende Bodensatz enthält grosse, zum Theil in fettiger Entartung begriffene Plattenepithelien, ausserdem Fettröpfchen und Fettkörnchenzellen.

Behufs mikroskopischer Untersuchung wurden aus der Leber 4 Stücke herausgeschnitten, und zwar:

No. I an den Grenzen des eigentlichen Tumors, woselbst ganz kleine Cysten mit unbewaffnetem Auge zu erkennen waren. Härtung in Alcohol abs.

No. II aus dem rechten Leberrand, einem für das blosse Auge cystenfreien Theil. Mäller'sche Flüssigkeit, dann Alcohol absolutus.

No. III mitten aus der Tiefe des Tumors, so dass dies Stück nicht ron der Leberoberfäche begrenzt wird. Alcohol abs.

No. IV aus der Gegend von No. I. Müller'sche Flüssigkeit und Alcohol abs.

Die mikroskopische Untersuchung ergab Folgendes: In den vom Stück No. I angefertigten, mit Pikro-Lithioncarmin gefärbten Scbnitten zeigt sich viel neugebildetes, sehr zellenreiches, junges Bindegewebe, welches sich keiner der von Charcot für die verschiedenen Arten von Lebercirrhose aufgesteliten, und als für die Ursachen derselben bedeutsam erklärten Verbreitungsformen des Bindegewebes in der Leber anschliesst, sondern bald die Grenzen de: Lobuli innehält, bald, dieselben durchbrechend, kleinere und grössere Parenchyminseln umgiebt. Manchmal ist es fiächenhaft so weit verbreitet, dass sehr viele Lobuli völlig verschwunden sind.

Der Uebergang des Lebergewebes in jenes gestattet sich verschieden. Selten grenzt er sich scharf $a b$, meist ist er ein ganz allnählicher. 
In dem Bindegewebe befindet sich ausser ziemlich weiten, aber wenig zahlreichen Arterien eine sehr grosse Zahl von Epithelsträngen, welche den Eindruck der, bei Cirrhose vorkommenden, neugebildeten Gallengänge machen. An vielen ist keine Lichtung zu erkennen, atch nicht im Querschnitt; andere dagegen besitzen Lichtungen, zum Theil sogar so weite, dass die Wand derselben von 30-40 Epithelzellen, beziehungsweise Kernen, umgeben wird, In diesem Fall sind sie mit seichten oder tieferen Ausbuchtungen versehen. Die meisten dieser Epithelstränge oder Kanäle verlaufen gerade, viele auch stark geschlängelt, ja selbst schleifenförmig; eine baumartige Verzweigung, wie sie bei Cirrhose so gewöhnlich besteht, ist selten, die von 0 rth und Anderen beobachteten netzförmigen Verbindungen fehlen völlig.

Das Epithel dieser Gebilde ist ein einschichtiges, platt-cubisches, mit sehr vielen Kernen. Im Querschnitt, vieler dieser zelligen Gebilde fehit, wie gesagt, eine Lichtung, in anderen ist sie eben wabrnehmbar.

Hie und da finden sich kolbig erweiterte, mit Lichtung versehene blinde*) Enden der Cylinder und ausserdem eine grosse Zahl runder, eiförmiger oder unregelmăssig gebuchteter Cystchen, welche, wie auch die Gänge, bezw. Stränge, stets ron mehr oder weniger verbreitetem, zellhaltigem Bindegewebe umgeben sind.

Die Fasern und Zellen des letzteren ordnen sich hinter dem Epithel einigermaansen parallel zur Wölbung der Cystchen, ohne den Eindruck einer wirklichen eigenen Wand zu machen, was auch für die Gänge gilt. Allmählich hört diese Ordnung auf und der Verlanf desselben zwischen den Neubildungen wird ein regelloser.

Eine der kleinen Cysten bat eine Wand von nur der halben Dicke eines Leberzellenbalkens (Fig. 5); man bekam daher zunächst den Eindruck, als ob sie sich frei innerhalb eines Acinus entwickelt babe, indess spricht der Umstand, dass sie mitten zwischen zwei Venae centrales gelegen ist, doch auch für einen Zusammenhang mit dem Bindegewebe der Glisson'schen Scheide. Frei im Lebergewebe ist also keine Cyste entstanden.

Die Epithelien der kleinen Cysteu baben dasselbe Aussehen, wie die der kleinen Gänge: Thr nicht körniger Leib bleibt in Pikro-Lithioncarmin farblos,

5) Ackermann ${ }^{35 * *)}$ bat auf Grundlage von Injectionspräparaten das Vorkommen blinder Endigungen der bei Cirrhose neugebildeten Gallengänge mit Entschiedenheit bestriiten. Für den vorliegenden Fall muss ich aber behaupten, dass wirklich blinde Enden und nicht etwa convexe Schlängelungen vorliegen, welche jenes Bild nur vortäuschen, Dabei will ich aber gleich darauf hinweisen, dass das in Rede stehende Bindegewebe, wie später zu erörtern, wahrscheinlich nicht das Ergebniss einer interstitiellen Hepatitis ist, wie es in Ackermann's Präparaten der Fall war.

*:*) Die neben Namen gesetzten Ziffern beziehen sich auf die Nummern der hinten beigefügten Literatur. 
ihre sehr dicht gelagerten Kerne sind von etwas wechselnder Grösse, durchschnittlich kleiner als die der Leberzellen; sie haben verschiedene, mehrseitige Gestalt, sind nicht deutlich bläschenförmig und färben sich dunkler, als die der Leberzellen.

Nach langem Suchen gelang es, an einigen der allerkleinsten Cysten die Einmündung eines Ganges mit demselben Epithel tadellos nachzuweisen. In dem einen Falle setzt sich die fast kugelrunde Cyste (Fig. 3), ohne dass der in sie einmündende Gang eine allmähliche Erweiterung zeigte, an denselben an, so dass eine ganz plötzliche Erweiterung der Lichtung eintritt. Diese Cyste ist nicht durch den Schnitt eröfnet und dabei so klein, dass man bei versebiedener Einstellung der Schraube nach einander sämmtliche, die Kugeloberfäche überziehende Epitbelien zu Gesicht bekommt.

In anderen Fällen zweigen sich gleich mehrere Cystchen hinter einander von den Gängen $a b$ (Fig. 4). Im Schnitt erscheinen die zwischen den einzelnen Ausbuchtungen des Ganges befindlichen Gewebstheile oft als ziemlich scharfe, nach der Mitte dosselben vorspringende Leisten (Fig. 4, a). Sind die Ausbuchtungen zahlreich, so kömen die wunderlichsten Formen entstehen. Besonders reich an solchen Hohlräumen ist Stück IV, dessen Schnitte sich, mit blossem Auge betracbtet, wie ein Netz ausnehmen.

In allen Schnitien des Stückes I liegt eine fast hanflomgrosse Cyste von etwas länglicher Gestalt unter der Leberkapsel. Ihre Wand ist auf der Seite der Leberkaysel folgendermaassen gebaut: In den tieferen Schichten der hier verdickten Kapsel liegen gleichmässig roth gefärbte, byalin aussehende, knorrige, theilweise stark wellig verlaufende Gewebsbalken, hie und da mit einigen Kernen untermischt; näher der Cyste folgen dann einige Leberzellenreihen mit schmaleren $Z$ wischenräumen als gewöhnlich; sodann Bindegewebe mit langen Kernen und scbliesslich einschichtiges platt-cubisches Epithel. Die Dicke der Wand beträgt auf dieser Seite etwa $\frac{1}{2} \mathrm{~mm}$. In ihr verlaufen einige der genannten kleinen Gänge parallel der Cystenoberfläche; ausserdem wird sie von einem etwas grösseren scbräg durchsetzt, der mit freier Lichtung in die Cyste einmündet. Auf der Leberseite ist eine derartige Schichtung der Wand nicht vorbanden. Hier geht das Lebergewebe in eine bald ganz schmale, bald breitere Schicht eines zarten, zellenreichen Bindegewebes über mit einzelnen eingesprengten Leberzellen, was besonders bemerkenswerth ist. Darauf folgt dann die Epithelschicht.

In einzelnen Cysten hat sich eine gelbe Masse abgelagert, von der sich wegen der nothwendigen Schonung des Sehnittes nicht entscheiden liess, ob sie aus Blut oder galligen Bildungen besteht.

An manchen Stellen (Fig. 6) - in viel grösserer Zahl in anderen Stücken - umgiebt eine eigenthümliche, gleichmässig rosa gefärbte Masse, welche sich bei starker Vergrösserung in ziewlich breite rothe Fasern auflöst, unbestimmt geformte, bald dem Faserstoff, bald dem Schleimgewebe ähnliche Gebilde. Die zarten Fasern derselben setzen sich in die rosafarbenen Massen fort, quer in sie eintretend. Hie und da liegen Kerne in ihnen, deren Herkunft sich wicht erweisen liess, die aber an die der Gallengangs- 
epithelien erinnern. Diese Gebilde sind anderwärts faserig und enthalten dann Bindegewebszellen, ja auch deutlich sichtbare gefüllte Blutgefässchen.

Die Leberzellen nehmen in Pikro-Lithioncarmin die bekannte gelbliche Farbe an; ihre Kerne sind verschieden gross.

Stück II.

Enthält auch Bindegewebe, welches sich aber von dem in I. gesehenen bedeutend unterscheidet. Denn:

1) ist es zellenarm, also altes fertiges Bindegewebe;

2) ist es nicht so regellos verbreitet, wie jenes, vielmebr ist es zu schmaleren, eben noch mit blossem Ange wahrnehmbaren Zügen geordnet, welche eine Verdickung der Glisson'schen Scheide darstellen, meist zwischen den Läppchen verjaufen, an zanchen Stellen aber auch Theile derselben, ja sogar einzelne Zellen umwachern und mit der etwas verdickten Leberkapsel zusammenhängen. Dieses Bindegewebe findet sich auch in der Tiefe dieser Lebergegend und ist deshalb wohl (im Gegensatz zu dem in No. I beobachteten, wie später zu besprechen sein wird) anf eine interstitielie Hepatitis zurückzuführen.

3) enthält es kaum einige der kleinen Gänge; die wenigen vorhandenen sind im Querschnitt mit Epithelien erfüllt.

4) sind auch wikroskopisch keine Cysten zu finden.

Die Leberzellenkerne färben sich in diesem Stücke im Allgemeinen nicht. Stück III.

Nur in wenigen der hier grossen Cysten, welche nie unter einander in Verbindung stehen, ist noch Epithel zu sehen. Natürlich ist wegen des gegenseitigen Druckes keine einzige Cyste mehr rund. Die ganze zwischen denselben belegene Gewebsmasse besteht aus Bindegewebe, welches näher der Oberfiäche der Cysten knorrige, wellig verlaufende Züge bildet, weiterhin lockerer wird, und vielfach Spalten und auch zellhaitige Schichten zeigt. An vereinzelten Stellen siebt man auffallender Weise noch wohlerhaltene Leberzellen, ausserdem heerdweise viele recht kleine Zellenstränge und geringe Eiweiterungen derselben; in der Wand einzelner Cysten auch gefüllte Blutgefässe. Schliesslich fiel in diesem Stücke das Vorkornmen krystallinischer, unregelmässiger, durchscheinender Tafeln innerhalb der Cysten auf; dieselben sind farblos und baben manchmal dunkle Körner in sich. Letztere verschwinden auf Zusatz von Salzsäure, sind also Kalkkönchen, die Tafeln selbst widerstehen der Salzsäure, geben aber auch mit concentrirter Schwefelsäure und Jod-Jodkalium keine Cholestearinreaction. Dagegen scheiden sich dabei auf einigen Tafeln Fettsäurekrystalle aus.

Stïck IV ist so reich an Hoblräumen, dass seine Schnitte netuförmig aussehen, gleichfalls am reichsten an jungetn Bindegerebe, und deshalb ara an Leberzellen.

Obwobl es nun mehr als wahrscheinlich war, dass die kleinen Gänge neugebildete Gallengänge seien, wurde an einigen Schnitten von Stück IV nach Gallenablagerungen in den Cysten gesucht. Es fanden sich dabei in einigen gelbe Massen wie in I, die mit der Gmelin'schen Salpetersäure- 
mischung eine grüne Farbe erzeugten, also als gallenfarbstoffhaltige Stoffe anzusehen sind. Mithin müssen die Cysten, also auch die mit ihnen in Verbindung stehenden Gänge mit absonderndem Lebergewebe in Zusammenbang sein. Auch diese Thatsache spriebt für ibre Natur als Gallengånge.

Das anatomische Ergebniss der Untersuchung ist also: In Theilen einer von interstitieller Entzündung befallenen Leber hat eine massenhafte Neubildung von Gallengängen stattgefunden mit cystischen Erweiterungen ihrer blinden Enden oder mit seitlichen Ausbuchtungen. Die Cysten sind ausser den kleinsten der kleinen bereits abgeschuürt.

Wann und wie erfolgte die Cystenbildung?

Nimmt man ein sehr langsames Wachsthum der Cysten an, so wäre vielleicht die Angabe der Frau, ihr Leib sei nach ihrer letzten, vor etwa 20 Jahren erfolgten, Entbindung stärker geblieben, auf beginnende Cystenbildung zu beziehen; will man nicht so weit zurückgreifen, so besteht doch sehr wahrscheinlich ein Zusammenhang der letzteren mit den vor 7 Jahren aufgetretenen Leberbeschwerden der Frau. Darnach wären die ältesten Cysten mindestens 7 Jahre alt. Der anatomische Befund erlaubt diesen Schluss durchaus; denn wenn die Cysten schnell gewachsen wären, so könnten sich im Stück III - dem der Mitte des Tumors entnommenen - mit dem vielen alten Bindegewebe und den dadurch gesetzten ungünstigen Ernährungsverhältnissen keine gesunden Leberzellen mehl vorfinden, was aber der Fall ist; vielmehr müssten sie längst zu Gruade gegangen sein.

Will man die Cysten in die allgemeine Ordnung der Cysten einreiheu, so ist der Gedanke an Gallenstaung als Ursache naheliegend. Und in der That sprechen verschiedene Verfasser von Gallenstaungscysten. So erwähnt Frerichs ${ }^{2}$ Erweiterungen an Gallengängen bei Cirrhose durch den Einfluss schrumpfenden Bindegewebes, ferner andere, in Folge von Verstopfung oder Zusammendrückung derselben entstanden. Er fügt hinzu: "Zuweilen entstehen auf diesem Wege sackförmige Hohlräume, Cysten, welche nach. allen Seiten geschlossen sind... Cruveilhier ${ }^{13}$ hat eine solche Anomalie abgebildet (Tome I. Livr. XII. pl. IV. fig. 3), leider ohne genaue Details über den Krankheitsfall beizufügen." Schliesslich bemerkt Frerichs noch, dass bei Schafen, welche an Distoma hepaticum leiden, ampullen- 
förmige Erweiterungen in Zusammenhang mit Gallengängen beobachtet worden seien.

Beschriebene Fälle sind meiner Kenntniss nach nicht häufig. Der von North ${ }^{14}$ ist nicht ganz beweisend für Staung, deutlicher scheint ein von Frarier ${ }^{27}$ in den Bulletins de la Société anatomique niedergelegter. Frarier fand bei völliger Versperrung des Duct. cysticus und choledochus die Lebergänge and ihre kleinen Aeste bedeutend erweitert; durch Aufschneiden der selben gelang es ihm, die Verbindung mit zahlreichen, in der ganzen Leber zerstreuten, bis haselnussgrossen Cysten nachzuweisen. Diese énthielten ein Gemisch von Galle und Eiter. Frarier spricht nicht ausdrücklich von Staungsscysten, setzt aber schweigend einen solchen Ursprung voraus.

Haben nun in unserem Falle die Cysten eine derartige Ursache? Beim Versuche des Nachweises stösst man sofort auf Schwierigkeiten. Denn es müsste der eine Staung bewirkende Verschluss, wegen der unregelmässigen Vertheilung der Cysten in der ganzen Leber, an zahlreichen verschiedenen Stellen eingetreten sein; eine Bedingung, für welche sich mangels vorhandener Steinbildung, Parasiten und schrumpfenden Bindegewebes kein Grund finden Jässt. Auch Epithelwucherung als Verschlussmittel fehlt.

Ferner sprechen gegen eine ursächliche Stauung in unserm Falle zwei Gründe, und zwar:

1. Die ganz plötzliche Erweiterung von Gängen (Fig. 1).

2. Das Vorhandensein scharf vorspringender Scheidewände (Fig. 4a) zwischen benachbarten, aus demselben Gange sich abzweigenden, Hohlräumen. Denn da sich in diesen Zwischenwänden nichts findet, was für besondere Widerstandsfähigkeit spricht, so müssten dieselben unter dem Drucke einer aufgestauten Flüssigkeit längst verschwunden oder wenigstens abgeplattet sein, wie die Nierenpapillen bei Harnstaung.

Ausserdem muss ich bekennen, dass mir die Bildung von Gallengangscysten durch Stauung überhaupt nicht recht fasslich erscheint. Da nehmlich nach unsern anatomischen Kenntnissen die interlobulären Gallengänge nicht blind enden, sondern als Gallencapillaren sich in's Innere der Läppchen hinein fortsetzen, so wären bei eintretendem Verschlusse eines Gallenganges zwar 


\section{3}

eine Erweiterung desselben, eine auch der kleineren in ihn einmündenden Aeste zu erwarten, nicht aber die Bildung blinder Hohlräume. Demnach möchte ich auch in dem oben erwähnten Falle von Frarier das Vorkommen von Gallengangscysten nicht ohne Weiteres als Folge des Verschlusses des Duct. cysticus ansehen, sondern halte eine Unabhängigkeit beider Erscheinungen nicht für ausgeschlossen.

von Recklinghausen ${ }^{1}$ hat in einer grossen Arbeit über die Entstehung von Schleimcysten sich auch über die der Flimmercysten in der Leber geäussert, welche Friedreich ${ }^{4}$, Eberth ${ }^{3}$ und er selbst beobachteten. Er führt die Cystenbildung auf eine vorhergehende Myxadenitis hyalinosa bezüglich fibrosa zarück, die nachweislich an einzelnen Stellen zu dauernder Versperrung führte. Die Erweiterung leitet er dann vom Fortbestehen der Absonderung des Schleimes seitens der Schleimdrüschen im Verein mit der ausserordentlichen Quellbarkeit des Schleimes ab.

In unserem Falle liegt die Sache anders. Die sich erweiternden Gänge sind neu entstandene, pathologisch, nicht physiologisch vorhandene; sie haben keine Schleimdrüsen und eine Myxadenitis fehlt.

Ueberhaupt sind wohl einzeln auftretende Cysten - von Recklinghausen, Eberth und Friedreich fanden nur je eine - von den massenhaften zu trennen.

Die vielfacb (Juhel-Rénoy ${ }^{8}$, Sabourin ${ }^{9}$, Courbis ${ }^{11}$, Bristowe ${ }^{20}$, Babinski ${ }^{26}$, Joffroy ${ }^{28}$, Leboucher ${ }^{29}$ u. A.) beschriebenen, gemeinsam vorkommenden Leber- und Nierencysten werden auf eine Anlage im Mutterleibe zurückgeführt. In unserem Falle waren die Nieren normal.

Von der Sabourin'schen ${ }^{10}$ Eintheilung in 1) eigentliche Gallencysten von grösseren Kanälen, 2) angiomatöse biliäre Cysten aus Kanälen, welche aus Leberzellbalken (bei Cirrhose) entstanden sind, 3) Cysten aus Vasa aberrantia, 4) Cysten aus Schleimdrüsen der Gallengänge, 5) mikroskopische Cysten durch noduläre Hyperplasie und Adenome - von dieser Eintheilung komme ich noch später auf No. 2 und 5 zurück.

Nach Ausschluss aller jener Ursachen, welche Cysten hervorzurufen im Stande sind, muss man die Möglichkeit, die 
Cysten seien ächte Neubildungen, Adenome, in Betracht ziehen. Für ihre Natur als solche spricht zunächst der Umstand, dass nach der per exclusionem geführten Beweisführung nichts anderes mehr übrig bleibt. Wichtiger ist aber das, nach der so äusserst früh erfolgenden Abschnürung, fortgesetzte Wachsthum der Gebilde. Ein so andauerndes Wachsen plegen wir ja als eines der Kennzeichen von Geschwülsten anzusehen. Auch auf die kleinen Gänge müssen wir den Namen "Geschwulst" anwenden. Wir haben zum Theil blind endende Gallengänge vor uns. Dieselben lassen sich, da normaler $W$ eise solche nicht vorhanden sind, nur als seitliche Auswüchse anderer auffassen, und verdienen somit den Namen "Sprossen" oder „Knospen". Diese Knospen sind dann theils massiv, ohne Lichtung, "Vollknospen", und bilden erst nach einer gewissen Zeit an ihren Enden einen sich später abschnürenden Hohlraum - oder sie erscheinen gleich von vornherein als "Hohlknospen“, Ausbuchtungen, welche tiefer und tiefer werden und sich schliesslich abschnüren. Es ist dies ein Vorgang, wie er sich z. B. auch bei der Bildung des Ovarialcystoms zeigt; bei diesen sind es die Drüsenschläuche, welche seitliche Sprossen treiben; diese werden dann zu Cysten.

Darnach würden unsere Cysten als Cystadenome der Gallengänge zu bezeichnen sein. Es liegen mehrere Beobachtungen über Adenome in der Leber vor. Betrachten wir einige näher des Vergleiches wegen!

Kelseb und Kiener ${ }^{36}$ haben 1876 in einer Arbeit über Adenome in diesem Organ eine Trennung derselben in zwei Arten vorgenommen und sie "adénomes hépatiques" und "adénomes biliaires" unterschieden. Sie fanilen (Observation I und II) knotige Leberzellenadenome, wie sie Griesing er und Rindfleisch sowie Eberth ${ }^{39}$ an einem in Zürich zur Beobachtung gekommenen Falle schon beschrieben hatten. Dieselben entstanden durch Sprossung von Leberzellenbalken oder durch allseitiges Wachstbum der Lobuli. Ihre Zellen zeigten Kernvermehrung und Vermehrung ihrer selbst durch Tbeilung, und die von ihnen ausgekleideten Kanäle bildeten ein unregelmässiges Naschenwerk: "Adénome hépatique".

Gleichzeitig fanden die beiden Forscher (in Observation II) in dem Bindegewebe der sehr heftigen Hepatitis auffallend viele neue Gallengänge. Gegabelt, netzartig verbunden, oft bedeutend erweitert, sprungfederförmig gewunden, verbreiten sich diese Röhren in vielen Verzweigungen um die in Atrophie begriffenen Lobuli, dringen sogar in's Innere derselben ein und setzen sich an die Stelle der Leberbalken. Sie finden sich auch zu Nestern 
vereinigt, in denen sie von einander durch Wände aus jungem Bindegewebe getrennt werden. Ihr einschichtiges Epithel hat in den kleinsten Gängen einen deutlichen Kern und wenig Protoplasma, in den grösseren Gängen ist das Epithel cubisch, in den grössten cylindrisch. Lichtungen sind kaum oder gar nieht zu erkennen.

In günstigen Schnitten ist der Uebergang von Leberbalken in diese Kanäle zu sehen und die allmäbliche Veränderung der Leberzellen (S. 647).

An auderen Stellen bilden dieselben kein Netz, sondern durchziehen regellos das Bindegewebe, welches an Stelle des völlig verschwundenen Lebergewebes getreten ist. Sie sind hier oft buchtig und mit seitlichen Ausläufern verseben und enden oft in einfache oder doppelte, gewöhnlich sehr erweiterte Blindsäcke. Ibr Epithel ist 1-3schichtig, cubisch oder cylindrisch. "Diese Zellen sind nichts Anderes, als die Epithelien von Gallengängen mittJerer und grösserer Weite." Kelsch und Kiener knüpfen daran die Bemerkung: „Wir sind versucht, den Namen „Polyadénomes biliaires“ auf diese eingekapseiten oder freiverbreiteten Baufen von Ausführungsgängen anzuwenden und eine gewisse Analogie, einen gewissen Parallelismus, zwischen der Vermehrung der absondernden Drüsentheile (Adénomes hépatiques) und der Nenbildung und knotigen Anbäufung der Abfuhrwege (adénomes biliaires) zu sehen."

Greenish ${ }^{34}$ wurde 1882 durch Befunde an 2 von 3 beobachteten Fällen von Adenomen in der Leber gleichfalls zur Trennung von Leberzelladenomen und Gallengangsadenomen gebracht. Ich lasse seine Beobachtung No. I im Auszuge folgen: Im rechten Leberlappen eines 6 ljährigen Mannes sass ein reichlich erbsengrosser, runder, scharf abgegrenzter, derber Knoten, vom Lebergewebe durch einen Bindegewebssaum abgetrennt; er besteht der Hauptmasse nach aus schmalen, vielfach gewundenen, verzweigten und unter einander zusammenhängenden Scbläuchen und Cylindern, eingebettet in Bindegewebe. Die Zellen derselben sind kaum halb so gross, wie die Leberzellen und gleichen am meisten denen der kleinen Gallengänge. Das Gesammtaussehen der zelligen Gebilde erinnerte gleichfalls sehr an das von Gallengängen. Epithel 1-6schichtig. Lichtung vorhanden oder fehlend. Am Rande der Geschwulst ausserdem Kanäle, die leicht als Gallengänge zu erkennen waren; sie setzten sich, wie vielfach zu sehen, in jene Gebilde fort, wobei sich ihre Zellen allmählich in die jener umformten. Benachbarte Leberzellen mit Fett infiltrirt.

In seiner Beobachtung II beschreibt Greenish in der Leber eines 75jäbrigen Weibes eine bohnengrosse, derbe, im Schnitt körnige, einer acinösen Drüse äbnliche Geschwulst. Sie bat eine Bindegewebskapsel und wird in Innern durch Bindegewebswände in Läppehen getheilt. Diese sehen im Schnitt verschieden aus, zum Theil erinnern sie weder in ihrer Form noch in der Anordnung und Beschaffenheit der Zellen an normales Lebergewebe oder äberhaupt an ein physiologisches Drüsengewebe.

Die Zellen erinnern in den Randgegenden der Läppchen noch am ehesten an Leberzellen, im Inneren durchaus nicht mehr. Man siebt einen allmäh- 
lichen Uebergang dieser in jene und ebenso einen Uebergang jener in die Epithelien von Cylindern, welche denen der Beobachtung I gleichen, somit also eine Fortsetzung von Gallengängen darstellen.

Green ish fügt binzu: „Diese Bilder sprechen dafür, dass auch in diesem Falle die neugebildeten Drüsenelemente aus den Gallengängen hervorgegangen sind, nur dass die Zellen zum Theil eine höbere Entwickelungsstufe erreicht haben, als im vorigen Falle." $Z u$ diesen Gebilden rechnet Greenish noch einen Fall, den Wagner ${ }^{38}$ (S. 473) beschreibt.

Birch-Hirschfeld ${ }^{33}$ lässt in seinem Lehrbuch S. 953 gleichfalls die Möglichleit der Adenombildung durch Wucherung des Gallengangepithels offen. Er sah in einer Leber massenhafte Knoten verschiedener Grösse, „zwischen ihnen an manchen Stellen umfängliche Wucherung schwieligen Bindegewebes. Hier nun ..... bedeutende Wucherung des Epithels der intraacinösen Gallengänge, durch welche dieselben zum Theil in ganz solide Zellmassen umgewandelt wurden; ferner fanden sich an denselben auch sinusartige Ausbuchtungen und solide Sprossen“.

Sabourin ${ }^{9}$ veröffentlichte 1882 Beobachtungen über gleichzeitig vorkommende Leber- und Nierencysten.

I. In einem Fall von "theilweiser eystischer Entartung der Leber und Nieren bei granulirter Cirrhose und Bright'scher Krankheit", fand Sabourin die Bindegewebszüge in der Leber voll alter und neuer Gallengänge. Die umschlossenen Lebergewebsreste erlitten oft in geschlossener Masse die Umbildung in solche. Die alten Gänge zeigten eine chronische, allgemeine Entzündung mit Verstopfung durch Epithelpfröpfe. Die neuen gingen sichtbar aus Leberzellenbalken hervor; einige schon erweitert. Epithel einschichtig. Ferner fand er einen Heerd kleiner Hohlräume in einer Form, die ihn zur Bezeichnung "angiomes biliaires" veranlasste, und schliesslich grosse einund mebrkammerige Cysten. Eine Einmündung von Gallengängen in diese hat er nicht gesehen, aber wegen der verschiedenen Weiten der Hoblränme nimmt er doch eine Entstehung der Cysten aus den Gallengängen an, wobei er die „angiomes biliaires" als ein Mittelglied betrachtet. Er stellt die Entstehungsweise der Cysten so dar: „In Leber und Nieren verliert durch das Vorhandensein der Cirrhose eine grosse Zahl der Drüsenbestandtheile alle "Caractères fonctionels", ihr Epithel kehrt durch eine Reihe von Veränderungen zum "état indifférent" zurück." Diese sollen dann, wie es die Regel bei der Cirrhose zu sein scheine, atrophiren. "Aber", fährt er fort, „durch eine Abweichung von diesem gleichsam normalen Vorgang, eine Abweichung, deren Ursache in ibrem Wesen uns entgeht, wird dieser Uebergangszustand der Zeitpunkt, in dem das Epithel .... auf eigne Faust wächst, ohne dass jrgend etwas im gestaltlichen oder mikrochemischen Verhalten bliebe, was an den früberen Zustand dieses Epithels erinnerte. Nur ibr Sitz kann beweisen, dass es früher arbeitendes Epithel der Leber und Nieren gewesen sei. “

Sabourin erklärt seine Cysten für „ächte cystische Epitbeliome“ und setzt sie den Polyadénomes von Kelsch und Kiener zur Seite. Er bält den Vorgang für "processus épithélial primitif“. 
Eine zweite Beobachtung eigte in dem cirrhotischen Gewebe viele neue Gänge und Massenumformung einer ganzen Gegend von Leberzellbalken in „pseudocanalicules biliaires", fft mit blinden Enden versehen, in denen bräunlich-grüne Klumpen lage. Ausserdem waren wieder einige Gallengangsangiome vorhanden, Cystn aber nicht; der Vorgang war also nicht so weit gedieben, wie in der erstn Beobachtung.

Indem er nun seinen Fall len Polyadénomes von Kelsch und Kiener anreiht, knüpft er folgenden ǰchluss über die Geschichte der gesehenen Epitheliome daran; er sagt: ,Wenn auch das regelmässige Schicksul der neugebildeten Kanäle die allmäliche Atrophie ist, so ergiebt sich aus dieser Gesammtheit von Thatsachen, lass wir einige Arten der Abweichung von dieser Atrophieentwickelung kemen:

1. Durch Wucherung ibresEpithels und Auswachsen desselben zu blinden Enden (cul-de-sac) können diese neuen Gallengänge zu Polyadénomes (Kelsch und Kiener) werden.

2. Durch ganz besondere Entwickelung ihres Epithels an der Oberffäche können diese nenen Kanäle der Entstehung angiomatöser Geschwülste voranfgehen, welche wegen ihrer 1bleitung von den Gallengangsnetzen den Namen Gallengangsangiome verdenen. Diese Veränderung sitzt mitten im cirrbotischen Gewebe.

3. Gallencysten "secundärer Erweiterung der Angiome".

Ein dritter Fall war wie der erste.

Die bisher beschriebenen und von mir angezogenen Fälle von Gallengangsadenomen haben zum Theil allerlei mit dem unsrigen gemeinsam, und doch deckt sich keiner völlig mit ihm. Am wenigsten ähnlich sind ihm die beiden Greenish'schen Fälle, weil ihnen die Cysten fehlen. Der von Birch-Hirschfeld kommt ihm näher, denn die "soliden Zellmassen zeigten auch sinusartige Ausbuchtungen". Kelsch und Kiener beschreiben Buchten, Vortreibungen, Schlängelungen der Röhren und schliesslich auch ,einfache oder doppelte, gewöhnlich sehr erweiterte, blinde Endigungen". Diese letzteren sahen wir auch in unserem Falle (Fig. 3 und 4) und fassten sie als kleinste, noch nicht abgeschnürte Cysten auf. Also etwas Gemeinsames!

Sabourin's Angiome fehlen freilich in unserem Falle, jedoch ist dies kein wesentlicher Unterschied, da das Bild eines Angiomes nur durch die grössere Menge erweiterter Gänge hervorgerufen wird.

Die Beobachtung von Kelsch und Kiener kommen also der unsrigen am nächsten.

$\mathrm{Zu}$ beachten ist ferner ein von $\mathrm{Hu}$ eter ${ }^{6} 1887$ beschrie- 
bener Fall. Auf der chirurgischen Kinik in Göttingen wurde einem 11 jährigen Müdchen eine, $2 \frac{1}{2} 1$ bräunlich-grauer Flüssigkeit haltende, Cyste von der unteren Lberfäche entfernt. Nachdem sie $1 \mathrm{Jahr}$ in Alkohol gelegen hatb, untersuchte sie Hueter in dem pathologischen Institut.

Wand bindegewebig, $\frac{1}{2}-4 \mathrm{~mm}$ dick, ungeben von einem sich verdïnnenden und allmählich aufhörenden Mantel pon Lebergewebe. In ibr sass eine zweite apfelgrosse, mit der grossen durh eine Oeffnung in Verbindung stehende Cyste, und eine ganze Anzahl kleierer befand sich in der Wand der grossen. Mikroskopisch ergab sich dasibst Verfettung der Leberzellen und eine Bindegewebswucherung von der Uregelmässigkeit der Verbreitung, wie in unserem Falle. An wenigen Stellen cyanotische Atrophie. Das Epithel der mittelgrossen (mikroskopischen) Cysien cylindrisch, das der grösseren mehr abgeplattet. Die Cysten waren rund der eiförmig, oft gebuchtet, er sab mit demselben Epithel ausgekleidete Ginge in sie einmünden, welche sich oft mit anderen gabelig vereinigten.

Der Befund ist also, abgesehen von der gleichgültigen Verschiedenheit des auskleidenden Epithels dem unsern wohl gleich. - Hueter selbst denkt an die Möglichkeit der Bezeichnung "Cystadenoma".

Zum Schlusse muss ich noch auf eine Beobachtung $\mathrm{Nau}$ nyn's ${ }^{24}$ eingehen.

Dieser fand in der ganzen Leber graue Fleckchen von höchstens Hirsekorngrösse, die auf Druck eine, völlig gewöbulicher Galle gleichende Flüssigkeit von sich gaben. Im mitroskopischen Schnitt zeigten die Geschwülstchen, welche in jungen Verdickungen der Glisson'schen Scheide lagen, viele Lücken. Dieselben haben selten runde oder eiförmige Gestalt, sind meist buchtig, indem Bindegewebspapillen in sie vorspringen. Sie stehen unter einander in Verbindung, sind von Pflasterepithel überzogen, „das auf's Genaueste dem der feineren Gallengänge gleicht", und enthalten gelbe formlose Massen. Hin und wieder liess sich im Schnitte die Einmündung von Gallengängen in die Cysten seben. (Die von Naunya beigefügte Zeichnung gleicht der unseren völlig.) „Man siebt dann in einzelnen Fällen das Lumen derselben sich plötzlich zu einem ziemlich umfangreichen Hoblraume erweitern, während das Epithel des Gallenganges in das die Wand des Hoblraumes auskleidende continuirlich übergeht. Der betreffende Gallengang scheint mit dieser Erweiterung stets zu enden, wenigstens war ein $Z$ usammenhang letzterer mit einew zweiten Gallengange niemals naçhzuweisen."

Ausser diesen Formen finden sich, gleiehfalls in Verbindung mit Gallengängen stehende Hohlräume mit vielen Ausbuchtungen, welche durch eigenes Wachsthum immer tiefer werden, während gleicbzeitig bindegewebige Wucherungen sie an einzelnen Stellen wieder verengern. So entsteht ein "System 
höcbst unregelmässiger, bald spaltartiger, bald gangartiger, unter einander vielfach communicirender Hoblräume". Hat die Geschwulst Mohnkorngrösse erreicht, so wächst sie nicht mehr "rein passiv unter dem Druck anfgestauter Galle", sondern durch knospenartige Epithelsprossen der Wand, welche in das Bindegewebe hineinwuchern, sich schliesslicb aushöhlen und mit der Mutterböble in Verbindung bleiben. Dieser Befund deckt sich bis auf die fehlende Abschnürung in allen Einzelheiten mit dem unseren.

Naunyn hat seinen Fall Cystosarcoma hepatis genannt; indess scheint es doch wohl gerechtfertigt, ihn dem Hueterschen und meinem anzureihen und die drei Neubildungen, vielleicht auch den Fall von $\mathrm{Kelsch}$ und $\mathrm{Kiener}$, als Gallengangscystadenome zu bezeichnen. Vielleicht gehört zu ihnen noch der eine oder andere Fall der beigefügten Literatur, z. B. der von Sabourin. Beweisend ist keiner.

Die Cysten erreichen, wie wir gesehen haben, durch andauerndes Wachsthum bedeutende Grössen. Es liegt dabei nahe der Gedanke an eine mögliche Rückbildung einer Anzahl derselben, welche etwa eine geringere Kraft der Ausbreitung besitzen. Zur Prüfung dieser Frage muss ich auf einen oben beschriebenen Befund eingehen und zwar auf die Gebilde, deren eines Fig. 6 zeigt.

Diese Dinge möchte ich für Schnitte von Hohlräumen halten, da die eigenthümlich hyalin aussehende Masse a im Schnitte einen völligen Ring darstellt, und da nach Anfertigung einer Reihe von Schnitten in dem letzten derselben diese Masse eine mehr flächenhafte Verbreitung hatte. Der Verdacht, sie stelle die veränderte Wand einer Cyste dar, ist naheliegend, und man könnte an einen Rückbildungsvorgang denken. Was aber bedeutet die eigenthümliche Füllmasse? Sie ähnelt, wie wir wissen, bald dem Faserstoff, bald dem Schleimgewebe, zeigt in anderen Schnitten Bindegewebe mit dentlichen Kernen, ja selbst gefüllte Gefässe, so dass man an einen fortschreitenden Vorgang, an das Bild einer Vene mit Organisation eines Thrombus erinnert wird. Nur liegt ein wesentlicher Unterschied gegenüber der Thrombenorganisation darin, dass die Cysten keinen einer Organisation fähigen Inhalt besitzen. Angenommen, es habe sich vor der Entwickelung dieser Füllmasse ein solcher in den Cysten nicht gebildet - und nichts spricht dafür, dass es geschehen sei -, so ist eine Entwickelung von Gewebe aus der Cystenwand her- 
aus, an welche man demnächst denken müsste, ein Vorgang, für den man vergeblich eine Analogie sucht.

Sabourin ${ }^{9}$ (S. 220 und 221) beschreibt bei Besprechnng der Cysten ähnliche Gebilde als "des tissus fibro-muqueux" und bildet sie auch ab. Er redet von einer Art Heilung und nenut diese Gebilde geradezu "Cystenleichname“. Ob diese Auffassung richtig ist, lässt sich jetzt noch nicht entscheiden, vielleicht später, wenn erst mehr Beobachtungen dieser merkwürdigen Gebilde vorliegen werden.

Eine weitere zu erörternde Frage ist die nach dem Zusammenhang zwischen der Entwickelung der Adenome und der des vorhandenen Bindegewebes.

Wir haben, wie bemerkt, in der vorliegenden Leber zwei Arten von Bindegewebe, welche sich in Beziehung auf ihre Verbreitungsform, ihr Alter, ihren Gehalt an Gallengängen und durch ihren Sitz im Organ deutlich von einander unterscheiden. Die eine Art verdankt einer Hepatitis interstitialis ihren Ursprung, ist alt und kommt überall da vor, wo Cysten fehlen; die andere besitzt im Gegensatze zu jener als Zeichen der Jugend eine grosse Menge von Zellen, und findet sich nur in der Umgebung der kleinen Cysten und Gallengänge, deren Gebiet sie nie weit überschreitet. Es muss also ein engerer Zusammenhang zwischen dem jungen Bindegewebe und der Adenombildung bestehen, als zwischen dem alten und diesen Gebilden. Daher muss man, so meine ich, bei Untersuchung der Frage nach dem Verhältniss zwischen der Entstehung des Bindegewebes und der der Adenome diese beiden Arten von Bindegewebe aus einander halten.

Was zunächst das alte, durch Hepatitis entstandene, betrifft, so scheint mir nicht viel Grund für die Annahme vorhanden zu sein, seine Entstehung habe die der Cystadenome bewirkt. 'Denn Cirrhose ist eine ebenso häufige Krankheit, wie die Adenome eine selten beobachtete sind. Allerdings sind Cysten $\left(\mathrm{Frarier}^{9}{ }^{9}\right.$ ) wie auch Adenome (Simmonds ${ }^{3 x}$ ) häufig gerade in cirrhotischen Lebern beobachtet worden; aber damit ist bei der verhältnissmässig geringen Zahl von Beobachtungen dieser epithelialen Gebilde, ein ursächlicher Zusammenhang zwischen beiden Krankheiten noch nicht erwiesen. Ich betrachte daher vorläufig die vorhandene Hepatitis und die Adenombildung als unabhängig von einander. 
Das junge Bindegewebe anlangend, meine ich ebenfalls, dass es nicht die Mutter der Adenome sei, sondern halte das umgekehrte Verhältniss für wahrscheinlicher. Wir finden ja überhaupt in der Leber eine Bindegewebsentwickelung als Antwort auf die mannichfaltigsten Reize.

So werden die Blasen des ein- wie des mehrkammerigen Echinococcus von Bindegewebe umgeben, und zwar die letzteren nicht nur in ihrer nächsten Nähe; bei Syphilis, bei Stauungen des Pfortaderblutes wie der Galle erscheint Bindegewebe. Für besonders wichtig aber halte ich wegen der Analogie der Fälle die Entstehung desselben im Gefolge epithelialer Vorgänge. Nach Orth ${ }^{16}$ können sich knotige Hyperplasien durch eine förmliche bindegewebige Kapsel von ihrer Umgebung abgrenzen. Eberth machte einen gleichen Befund in dem GriesingerRindfleisch'schen Falle von hyperplastischen Knoten der Leberzellenbalken; die grösseren derselben waren von einer zarten Kapsel jungen Bindegewebes umgeben. Kelsch und Kiener ${ }^{36}$, Greenish ${ }^{34}$, Willigk ${ }^{41}$, Jungmann ${ }^{37}$ sahen Aebnliches. Nach diesen Beobachtungen erscheint die Behauptung, die Gallengangsentwickelung sei die Ursache des Hervorspriessens jungen Bindegewebes, als durchaus zulässig. Sie erhält noch eine Stütze in dem Umstand, dass mit zunehmendem Alter der Cysten auch das Bindegewebe an Masse zunimmt.

Wir kommen nun zur Frage nach dem Ursprunge der Gallengänge. Ueber den Ursprung solcher Gänge sind, soweit die bei Cirrhose und acuter Leberatrophie neugebildeten in Betracht kommen, die Pathologen verschiedener Ansicht. Seit längerer Zeit nahmen viele, besonders Klebs an, sie seien nichts als veränderte Leberbalken, andere dagegen erklärten sie für Gallengänge, seitdem ihr Zusammenhang mit den interacinösen Gallengängen nachgewiesen war (Waldeyer, Cornil), und behaupteten meist, sie entständen durch Knospung aus diesen.

Nun beobachteten Charcot und Gombault, welche zusammen über Gallengänge arbeiteten, den Zusammenhang der fraglichen Gebilde nicht nur mit den normalen Gallengängen, sondern auch „à plein canal" mit den Leberzellenbalken, wobei sich die Zellen letzterer allmählich umwandelten.

$\mathrm{Kelsch}$ und Kiener ${ }^{33}$ beschrieben in einer gleichzeitig ge- 
machten, aber später, als der Aufsatz von Charcot und Gombault, veröffentlichten Arbeit dasselbe, und gaben sogar eine vortreffliche Abbildung des kegelförmigen Mittelstückes, auf dem sich eine Vermehrung und Umwandlung der Leberzellen und eine Verjüngung der Leberbalken in die dünneren Gallengänge vollzieht.

Ackermann ${ }^{35}$ hat auf Grund von Injectionspräparaten die Richtigkeit derartiger Angaben bestritten, indess kommt Orth neuerdings auf jene zurück und bildet eine solche Stelle ab ( ${ }^{16} \mathrm{Bd}$. I Fig. 196), ohne die Entstehung durch Knospung füŕ unmöglich zu halten.

In unserm Falle muss, trotzdem die Neubildung hier muthmaasslich nicht durch Cirrhose bewirkt ist, auch eines von beiden stattgefunden habea, da ein drittes nicht denkbar ist. In der That sind bei Adenombildung und bei primärem Lebercarcinom beide Entwickelungsarten beobachtet worden. Jung$\operatorname{mann}^{37}$ sah in einem Falle von Adenom, welches in Gestalt zahlreicher neugebildeter Gallengänge auftrat, stellenweise eine unmittelbare Fortsetzung derselben in Leberzellenbalken. Na un y ${ }^{42}$ trommt durch Beobachtung primärer Leberkrebse zu dem Schluss (S. 730), „dass sich die Epithelzellen der Gallengänge durch Wucherung an der Bildung von Geschwülsten betheiligen, die zweifellos als Carcinome anzusehen sind. Jie Zellen der Neubildungen - fährt er fort - von epithelialem Charakter, die sogenannten Krebszellen, gehen jedenfalls nicht selten aus einer Wucherung der Gallengangsepithelien hervor; ob auch die Leberzellen sich an der Wucherung betheiligen, gelang mir nicht mit Sicherheit festzustellen; die Beobachtung des Ueberganges des Neoplasmas in das normale Leberparenchym schien dafür zu sprechen." Waldeyer ${ }^{43}$ bestätigt Naunyn's Beobachtung. Perls ${ }^{44}$ sah den Uebergang von „durch Bindegewebswucherung abgeschnürten Leberzellhaufen zu Carcinomalveolen" ganz deutlich.

Es sind also in der That bei diesen epithelialen Neubildungen beide Entstehungsarten beobachtet worden.

In unserm Falle sehen wir Knospung in Gestalt solider Epithelstränge, und zwar ancheinend nur an neugebildeten Gallengängen. Ob auch eine Umwandlung von Leberzellen stattgefunden hat, ist nicht genau zu sagen. Im Stück IV habe ich 
zwar an einzelnen Stellen, wo die Grenze zwischen Binde- und Lebergewebe verhältnissmässig scharf war, in diesem Grenzgebiete wiederholt Zellenstränge gesehen, deren Aussehen für eine solche Umwandlung sprach. Ihre Zellen waren nehmlich kleiner als Leberzellen, ähnelten also in der Beziehung den Gallengangsepithelien; wiederum aber unterschieden sie sich von letzteren durch die gelbliche Farbe ihres Leibes. Ihr Aussehen hat also von dem beider Zellenarten etwas. Da ich aber trotz langen Suchens Uebergänge dieser Stränge in Leberzellenbalken nicht gefunden habe, so muss ich diese Frage offen lassen.

Bezïglich der Adenomentwicklung haben wir keine Ursache gefunden. Es muss demnach, wie so oft, genügen, den Fall in eine Gruppe von Erscheinungen einzureihen, deren Bau und sichtbare Wachsthumsvorgänge uns bekannt sind, wenn uns auch die Ursachen der letzteren entgehen.

Herrn Professor Orth, meinem hochverehrten Lehrer, welcher in liebenswürdigster Weise diese Arbeit leitete, erlaube ich mir für seine Güte und Unterweisung den innigen Dank des Lernenden auszusprechen.

Figur 3 und 5 sind bei 340 facher, Figur 4 und 6 bei 160 facher Vergrösserung gezeichnet.

\section{L i t e r a t u r.}

1. von Recklinghausen, Ueber die Ranula, die Cyste der Bartholin'schen Drüse und die Flimmercyste der Leber. Dieses Archiv Bd. 84. S. 425 .

2. Frerichs, Klinik der Leberkrankbeiten.

3. Eberth, Cyste mit Flimmerepithel in der Leber. Dieses Archiv Bd. 35. S. 478.

4. Friedreich, Cyste mit Flimmerepithel in der Leber. Dieses Archiv Bd. 11. S. 466.

כ. Davaine (Ueber multiple Lebercysten), Gazette des Hôpitaux. 1852, und Schmidt's Jahrbücher') Bd. 75. S. 282.

6. Hueter, Ein grosses Cystom der Leber. Göttinger Diss. 1887.

1) Sind 2 Bücher angeführt, so enthält das zweite nur ein Referat. 


\section{4}

7. Bouchut (Lebercysten), Gazette des Hôpitaux. 1872, und Schmidt's Jahrbücher Bd. 158. S. 248.

8. Juhel-Rénoy, Observation de dégénérescence kystique du foie et des reins. Révue de medeciue. Paris 1881, p. 929 , und Centralblatt der med. Wissensch. 1882. S. 398.

9. Sabourin, Contribution à l'étude de la dégénérescence kystique du foie et des reins. Archives de physiologie normale et pathologique. 1882. p. 63, und Centralbl. f. d. med. Wissensch. 1882. S. 760.

10. Sabourin, A propos de deux kystes du foie d'origine biliaire. Le progrès médical. Paris 1884 . No, 20 .

11. Courbis, Note sur une dégénérescence kystique du foie et des reins. Lyon médical. 1877. No. 16, und Jabresberichte von VirchowHirsch. 1878. I. S. 239.

12. Cruveilhier, Anat. pathol. générale. II. p. 835 .

13. Cruveilbier, Anat. path. du corps humain. Paris 1829-1835. Tome I. Livraison XII. p. 4 . fig. 3.

14. North, Case of a cystic tumour of the liver, caused by impacted gallstone in common bile-duct. New-York Medical Records. 1882.

I. p. 344 und Virchow-Hirsch's Jahresber. II. S. 179.

15. Virchow, dieses Archiv Bd.I. S. 114. Anmerkung.

16. Orth, Lehrb. d. spec. path. Anat. 1887. Bd. I.

17. British medical Journal. 1874. p. 700 (Operation einer Lebercyste) und Centralb]. f. Chir. 1875. S. 176.

18. Rokitanski, Lehrb. d. path. Anat. Wien 1861. Bd. 3. S. 263.

19. Wilks, Colloid diseases of the liver. Transactions of the Pathological Society of London. 1859. Vol. 10. p. 178.

20. Bristowe, Association of cystic disease of the liver and kidneys.

Transact. of the path. Soc. London 1859. T. 10, p. 174.

21. Hare, Transact. of the path. Soc. Lond. 1859. T. 10. p. 182.

22. Bristowe, Cystic disease of the liver, associated with similar disease of the kidneys. Ibidem. 1856. T. VII. p. 229.

23. Virchow, D. kraukhaft. Geschwülste.

24. Naunyn, Ueber eine eigenthümliche Geschwulstform der Leber (Cystosarcoma bepatis). Müller's Arch. 1866. S.710.

25. Baraduc, Kystes du foie. Bulletins de la Société anatomique de Paris. 1876. p. 247.

26. Babincki, Kystes multiples du foie et des reins. Bullet. de la Soc. anat. Paris 1882. p. 341.

27. Frarier, Hépatite avec dilatations des canaux biliaires, consécutive à une atrésie du canal choledoche. Ibidem. 1866. p. 56.

28. Joffroy, Dégénérescence kyst. des reins et du foie. Ebend. 1868. p. 231.

29. Leboncher, Kystes nombreux du foie et des reins. Ebendas. 1869 . p. 243 .

30. Calot, Dilatations des voies biliaires. Kystes purulents du foie. Ebendas. 1870. 
31. Simmonds, Die knotige Hyperplasie und das Adenom der Leber. Arch. f. klin. Med. 1884. Bd. 34. S. 388.

32. Kiener et Kelsch, Note sur la néoformation de canalicules biliaires dans l'hépatite. Archives de physiol. norm. et pathol. Paris 1876. p. 778 .

33. Birch-Hirschfeld, Lehrb. d. path. Anat. 1877. S. 953.

34. Greenish, Ueber d. Adenom d. Leber. Wiener med. Jahrbücher. 1882. S. 411 .

35. Ackermann, Ueber die Neubildung von Gallengängen in der menschl. Leber. Sitzungsberichte d. naturforsch. Gesellsch. Halle, 28. Febr. 1880.

36. Kelsch et Kiener, Contributions à l'bistoire de l'adénome du foie. Arch. de phys. norm. et path. Paris 1876. p. 622.

37. Jungmann, E., Ein Fall von cirrhot. Leber mit Adenombild. und Uebergang in Carcinom. Diss. Berlin 1881.

38. Wagner, E., Drüsengeschwulst der Leber. Archiv der Heilk. 1861. S. 473 .

39. Eberth, D. Adenom der Leber. Dieses Arch. 1868. Bd. 43. S. 1.

40. Friedreich, Ueber multiple knotige Hyperplasie der Leber und Milz. Dieses Arch. 1865. Bd. 33. S. 48.

41. Willigk, Beitrag zur Histogenese des Leberadenomes. Dieses Arch. 1870. Bd. 51. S. 208.

42. Lancereaux, Contribution à l'étude de l'hépato-adénome (adénome hépatique) etc. Gazette médicale de Paris. 1868. Bd. 23. Lieferung 45 und 50 .

43. Naunyn, Ueber d. Entw. der Leberkrebse. Müller's Arch. 1866. S. 717 .

44. Waldeyer, Die Entw. d. Carcinome. Dieses Arch. 1872. Bd. 55. S. 218.

45. Per]s, Zur Histologie d. Lebercarcinoms. Dieses Arch. 1872. Bd. 56. S. 448 . 